\title{
Phaseless Characterization of Broadband Antennas
}

\author{
Ana Arboleya, Jaime Laviada, Juha Ala-Laurinaho, Yuri Álvarez, Senior Member, IEEE, \\ Fernando Las-Heras, Senior Member, IEEE, and Antti V. Räisänen, Fellow, IEEE
}

\begin{abstract}
A new efficient method for broadband antenna characterization from phaseless acquisitions in the frequencydomain is presented. The phase-retrieval technique is based on an extrapolation of the off-axis indirect holography. In common with the conventional approach, the power of the interferometric field of the antenna under test and a reference antenna, whose field is known in advance, as well as the power of the antenna under test alone, is measured at the desired frequencies. Nevertheless, the phase retrieval is accomplished independently at each spatial point by filtering in the time-domain rather than in the $k$-space. Thus, the dependency of the phase retrieval on the position accuracy is reduced and it can be accomplished simultaneously at all frequencies without resorting to iterative schemes. Moreover, it yields a less dense sampling and a phase retrieval algorithm not dependent on the geometry of the acquisition. The method is illustrated with a numerical example in the $W$-band as well as with two near-field measurement examples in the $K_{a}$ - and $W$-bands.
\end{abstract}

Index Terms-Antenna Measurement, Diagnostics, Phaseless, Broadband, Off-axis Holography, Leith-Upatnieks.

\section{INTRODUCTION}

B ROADBAND antennas have attracted great attention in the last years due to their applications in fields such as radar, multichannel frequency communications, Ultra Wide Band systems, etc.

Different measurement techniques have been developed in order to accurately characterize broadband antennas; these methods can be classified fundamentally into time-domain (TD) or frequency-domain (FD) methods [1].

There are different approaches to perform measurements in the TD, being pulse generator based systems the main one [2][4]. The dynamic range of these systems may not be sufficient, especially if network analyzers with TD modules are used to generate the signals. Thus, the distance between the Antenna Under Test (AUT) and the probe antenna has to be reduced [3]. Specific formulation for planar near-field (PNF) measurements in the TD has been developed [5], [6] alleviating this problem.

This work has been partially supported by the European Science Foundation under the RNP NEWFOCUS programme; by the Ministerio de Ciencia e Innovación of Spain /FEDER under project MIRIIEM-TEC2014-54005-P and TEC2014-55290-JIN; by the Gobierno del Principado de Asturias through the PCTI 2013-2017 GRUPIN14-114 and by predoctoral grant BP11-169; by grant LINE 525-002; and by the Academy of Finland through the DYNAMITE project.

A. Arboleya, J. Laviada, Y. Álvarez and F. Las Heras are with Departamento de Ingeniería Eléctrica of Universidad de Oviedo, Gijón E-33203 Spain (email: aarboleya@tsc.uniovi.es, jlaviada@tsc.uniovi.es, yalopez@tsc.uniovi.es, flasheras@tsc.uniovi.es).

J. Ala-Laurinaho and A.V. Räisänen are with the Department of Radio Science and Engineering and MilliLab, Aalto University School of Electrical Engineering, Espoo FI-00076, Finland (e-mail: juha.ala-laurinaho@aalto.fi, antti.raisanen@aalto.fi).

Manuscript received Month day, year; revised Month day, year.
Main advantages of TD measurements are: a) the reduction of the reflections caused by mismatch and multipath by means of time gating techniques, b) simple equipment setup, and c) efficiency, as the antenna can be characterized for all the frequencies from one spatial acquisition [2].

On the other hand, these methods present some disadvantages such as: a) excessive acquisition time for complete three dimensional characterization of antennas, b) presence of ringing patterns in the TD for narrow-band antennas and c) reduction of the signal-to-noise $(\mathrm{S} / \mathrm{N})$ ratio and, consequently, the measurement accuracy, caused by the decay of the pulse spectrum at higher frequencies.

The previous methods in the TD require acquisitions of amplitude and phase data, at least for near-field (NF) measurements, to compute the far-field (FF) pattern. The instrumentation needed for those complex acquisitions is more expensive than the one required for phaseless acquisitions.

Additionally, phase acquisition can be complicated at mmand submm-wave frequencies or in environments with poor thermal stability [7], [8]. Furthermore, errors due to cable flexing [9] or the accuracy of the positioners [10] become more relevant at higher frequencies.

FD techniques are the most employed for conventional antenna measurement [7]-[13]. Moreover, several methods based on wave expansions or equivalent currents for different acquisition geometries have been developed [14]-[16] for NF and $\mathrm{FF}$ acquisitions.

The main advantage of FD antenna characterization is the high accuracy and the number of the available techniques. On the other hand, broadband antenna analysis with conventional FD techniques could be very time-consuming for a high number of frequencies as data have to be independently acquired and processed for each of the analyzed frequencies.

Opposite to the TD techniques, several phaseless antenna characterization methods have been developed in the FD. These methods are divided into two main families: iterative and interferometric phase-retrieval methods.

Iterative methods are based on the acquisition of amplitude data in at least two independent surfaces [16], [17]. The phase of the field in each surface is retrieved after an iterative process. Those systems are very popular because they involve minor changes in the measurement system, nevertheless, main disadvantages are the stagnation problems and the high acquisition time, as two or more acquisition surfaces are needed.

Interferometric phase-retrieval techniques are based on the knowledge of a reference field (amplitude and phase) that is combined with the AUT field, creating an interference pattern that makes possible the retrieval of the phase of the AUT in the spatial frequency domain [18]-[23]. These interferometric techniques were originally introduced by Gabor in the optics 
field [24] and adapted to work with an off-axis reference signal by Leith and Upatnieks [25], thus are usually referred to as Leith-Upatnieks or indirect off-axis holography.

Accuracy of these indirect holography methods can be limited by errors in the characterization of the reference source and the overlapping between the spectra of the reference antenna and the AUT. A complete analysis of error sources can be found in [7], nevertheless the use of synthesized reference sources and modified holograms allows for an error reduction and a better performance of the phase-retrieval algorithms [22].

Other authors have developed hybrid methods based on the use of a modified probe [26]. This probe is composed by two identical antennas placed at a fixed distance and interfering each other through a microstrip circuit. The phase retrieval is performed by means of a minimization procedure of the phase difference between both probes for all the frequencies. Although the hybrid method can be applied to wideband antenna characterization, it requires a fixed sampling rate determined by the separation of the probes. Besides, the use of non linear minimization may suffer from local minima stagnation [27]. Separation of the antennas must be larger than half a wavelength to avoid coupling, hence several acquisition subsets have to be combined. Thus, the quality of the positioning, which is a handicap at mm-wave band and above, is expected to have an impact in the final result.

The proposed technique is a FD method capable of phaseless broadband antenna characterization. As it is based on offaxis holography, it is necessary to perform two amplitude-only spatial acquisitions to obtain the hologram and the squared amplitude of the AUT, providing the reference antenna is previously characterized.

In conventional off-axis holography, the phase is retrieved simultaneously for all the points of the measurement grid, after finishing a complete spatial acquisition, by filtering one of the terms of the hologram in the $k$-space. After that, the operation is repeated for each frequency. In contrast, for the proposed method, the phase is retrieved in the following way: for each spatial point in the measurement grid, a frequency sweep is performed and the hologram is obtained in the FD. Then, the phase retrieval is done, simultaneously for all the frequencies at that specific grid point, after filtering the desired term of the hologram in the TD and removing the effect of the reference antenna (a detailed explanation is given in Section II).

Main advantages with respect to the conventional method are listed next: 1) The phase of the AUT is retrieved point-bypoint hence the accuracy of the positioners is not as relevant as in conventional techniques, and the method can be applied to $\mathrm{mm}$ - and submm-wave bands antenna characterization with reduced uncertainties regarding the mechanical errors of the system [10], although accuracy is still necessary to perform the NF-FF transformation if no probe positioning correction algorithms are to be applied. 2) The technique is not dependent on the geometry of the acquisition either and therefore, nonredundant sampling techniques can be directly applied [20] to considerably reduce the number of acquisition points. 3) Overlapping of the terms of the spectrum of the hologram can be easily controlled by increasing the physical length of the reference antenna branch (see Fig. 1) and sampling

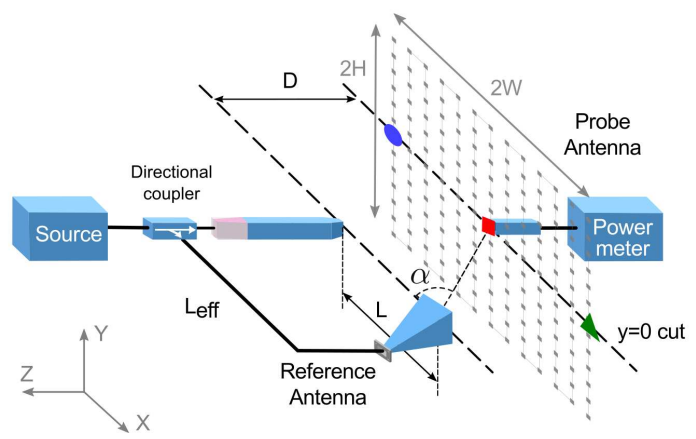

Fig. 1. Off-axis holography scheme for broadband antenna measurement. The holograms will be studied in the indicated points of the acquisition plane.

requirements are the same as the ones required in complex acquisitions with amplitude and phase, that is $\lambda / 2$ [14]. In the conventional off-axis holography the sampling has to be at least $\lambda / 4$ [21]-[23] or even denser and it might also be needed to resort to the use of phase shifters, which are challenging at high frequencies, for less directive antennas to reduce overlapping.

The herein presented technique is developed for a PNF acquisition although it can be directly applied to FF acquisitions and used in other geometries.

This paper is structured as follows. The proposed phase retrieval technique is explained in Section II. A simulation example of a broadband antenna in the $W$-band is shown in Section III. Two measurement setups in the $W$ - and $K_{a^{-}}$ band are presented in Section IV. Finally, main conclusions are drawn.

\section{METHOD}

The basis of the method for the point-by-point phase retrieval in the TD is developed in Section II-A. After the method is explained, the setup limitations regarding the selection of the main parameters such as the frequency sampling or the position of the antennas and the measurement steps will be addressed in Section II-B and Section II-C respectively.

\section{A. Off-axis holography extrapolation for broadband phaseless antenna characterization}

For the proposed technique, the physical arrangement of the elements is identical to the one in conventional offaxis schemes (see Fig. 1). The setup would benefit from the use of a variable attenuator or a variable gain amplifier to level the power of both antenna branches and increase the dynamic range of the system [21]. Keeping a constant dynamic range of the hologram as well as completely illuminating the acquisition plane with the reference signal is mandatory for a low error in the phase retrieval algorithm.

At each point of the acquisition plane the field is acquired along a set of equally spaced frequencies giving an interferometric signal:

$$
\begin{array}{r}
H(\vec{r}, w)=\left|E_{\text {aut }}(\vec{r}, w)+E_{\text {ref }}(\vec{r}, w)\right|^{2}= \\
\left|E_{\text {aut }}(\vec{r}, w)\right|^{2}+\left|E_{\text {ref }}(\vec{r}, w)\right|^{2}+ \\
E_{\text {aut }}(\vec{r}, w) E_{r e f}^{*}(\vec{r}, w)+E_{\text {aut }}^{*}(\vec{r}, w) E_{r e f}(\vec{r}, w),
\end{array}
$$


being $\vec{r}$ the position vector defining the position of the probe antenna in the acquisition plane and $w$ the angular frequency. The asterisk indicates the complex conjugate. $E_{\text {aut }}$ is the field radiated by the AUT and $E_{r e f}$ is the field radiated by the reference antenna, noting that both, $E_{\text {aut }}$ and $E_{\text {ref }}$, refer to the field component received by the probe antenna. The acquisition process can be repeated for the other component of the field just by changing the polarization of the AUT.

Then, by considering only one of the outputs of the directional coupler at a time, the square amplitude of the AUT and the reference antenna can be separately characterized and the modified hologram can be computed as:

$$
\begin{array}{r}
H_{m}(\vec{r}, w)=H(\vec{r}, w)-\left|E_{a u t}(\vec{r}, w)\right|^{2}-\left|E_{\text {ref }}(\vec{r}, w)\right|^{2} \\
\quad=E_{\text {aut }}(\vec{r}, w) E_{\text {ref }}^{*}(\vec{r}, w)+E_{\text {aut }}^{*}(\vec{r}, w) E_{\text {ref }}(\vec{r}, w) .
\end{array}
$$

The inverse Fourier Transform (FT) of the modified hologram produces the following signal in the TD, which has to be obtained at every point of the $2 \mathrm{~W} \times 2 \mathrm{H}$ measurement grid:

$$
\begin{array}{r}
h_{m}(\vec{r}, t)=e_{a u t}(\vec{r}, t) \otimes e_{r e f}^{*}(\vec{r},-t)+ \\
e_{\text {aut }}^{*}(\vec{r},-t) \otimes e_{r e f}(\vec{r}, t) .
\end{array}
$$

where $\otimes$ denotes a convolution, and $e_{\text {aut }}$ and $e_{\text {ref }}$ represent the inverse FT of the radiated fields.

A schematic representation of the modified hologram in the TD is shown in Fig. 2. The hologram is composed of the two cross-correlation terms thus, to retrieve the amplitude and phase of the AUT, it is necessary to filter the term corresponding to the convolution of $e_{a u t}(\vec{r}, t)$ and $e_{\text {ref }}^{*}(\vec{r}, t)$ as indicated in (4) providing no time overlap is present.

$$
h_{\text {mfiltered }}(\vec{r}, t)=\left\{e_{\text {aut }}(\vec{r}, t) \otimes e_{\text {ref }}^{*}(\vec{r},-t)\right\} R\left(t_{1}, t_{2}\right) \text {, }
$$

wherein $R\left(t_{1}, t_{2}\right)$ represents the rectangular time window from $t_{1}$ to $t_{2}$. The parameter constraints that allow to perform a correct filtering will be discussed in the next section.

Once the signal has been filtered in the TD, last step, as in the conventional off-axis approach, is the removal of the effect of the reference antenna back in the FD:

$$
E_{\text {aut }}(\vec{r}, w) \simeq \frac{H_{m \text { filtered }}(\vec{r}, w)}{E_{r e f}^{*}(\vec{r}, w)}
$$

The error of the phase retrieval algorithm will depend mostly on the separation of the cross-correlation terms in the TD. This separation is determined by the starting times of the signals coming from the AUT and the reference antenna, $t_{a u t}$ and $t_{r e f}$, and from their spread or duration, defined as $\Delta \tau_{a u t}$ and $\Delta \tau_{r e f}$, as it can be seen in Fig. 2 , and it will be addressed in the following section.

\section{B. Setup limitations}

According to the modified hologram scheme depicted in Fig. 2 two conditions can be established: a) time overlap can be avoided if the first condition of (6) is satisfied for all the sampling points, and b) frequency sampling must fulfill the expression in (7) [14].

$$
\left\{\begin{array}{ll}
t_{\text {aut }}-t_{\text {ref }}>0 & \text { original hologram } \\
t_{\text {aut }}-t_{\text {ref }}+\Delta \tau<0 & \text { swapped terms hologram }
\end{array},\right.
$$

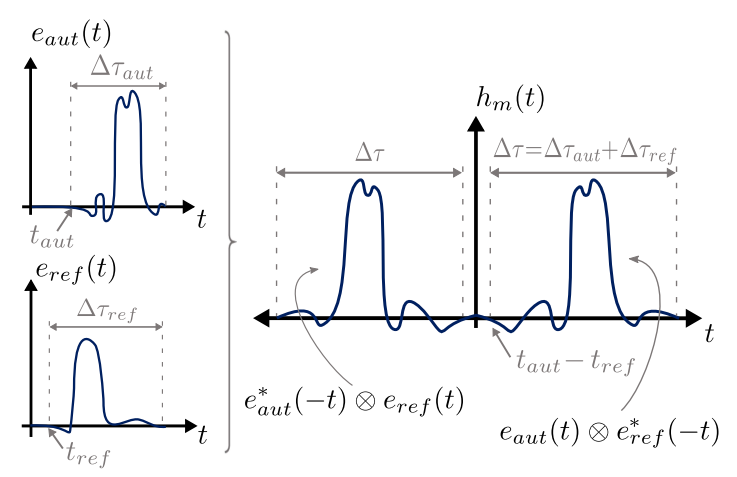

Fig. 2. Scheme of the AUT and reference signals in the TD and composition of the hologram for a specific point of the acquisition plane.

$$
\Delta f<\frac{1}{2 T}=\frac{1}{2\left(\Delta \tau+t_{\text {aut }}-t_{r e f}\right)},
$$

being $T$ the duration of the signal.

The first condition, given in (6), can be used to determine whether the position of the antennas and the size of the acquisition plane allow for a correct separation of the spectral terms. Then, from the second condition, given in (7), the minimum frequency sampling that guarantees that all the requirements are met can be obtained.

Since $t_{\text {aut }}$ and $t_{\text {ref }}$ represent the time that takes the radiation to travel from the reference antenna and AUT to the probe antenna, the previous constraints can be expressed as a function of distance.

Considering a symmetric acquisition plane in relation to the position of the AUT, and regarding the first requirement, as detailed in the first part of (6), worst case scenario corresponds to one of the corners of the acquisition plane on the side closer to the reference antenna ( $\Delta$ point in Fig. 1 for an horizontal cut).

The distance from the source to one of these corners (upper or lower corners of the acquisition plane corresponding to the worst case scenario) can be expressed as follows, neglecting the dispersion that can be introduced by the directional coupler and the antennas:

$$
\sqrt{D^{2}+W^{2}+H^{2}}>\sqrt{D^{2}+(W-L)^{2}+H^{2}}+L_{e f f}
$$

where $W$ and $H$ are the half width and the half height of the acquisition plane respectively, $D$ is the distance from the aperture plane of the antennas to the acquisition plane, $L$ is the distance between the center of the apertures of the AUT and reference antenna and $L_{e f f}$ is the effective length of the transmission line connecting the directional coupler and the reference antenna.

The upper limit for the previous condition when $W$ and $H$ go to infinite is given by:

$$
L_{e f f}<L
$$

The previous condition is expected to be never fulfilled as the geometrical arrangement of the antennas forces $L_{e f f}$ to be larger than the separation between antennas.

The effective length of the transmission line can be increased (and so $t_{r e f}$ ) until both cross-correlation terms are 


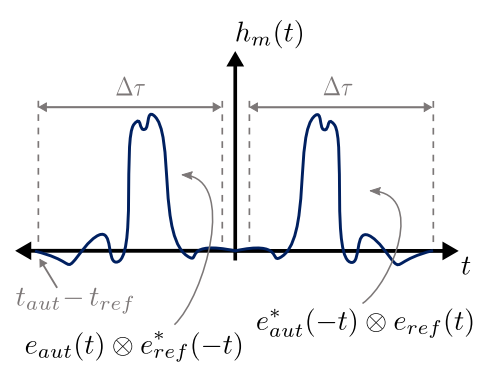

Fig. 3. Time-domain modified hologram with swapped cross-correlation terms.

swapped (see Fig. 3). In this case, the second condition in (6) has to be fulfilled, yielding the following expressions as a function of distance:

$$
\begin{gathered}
\sqrt{D^{2}+W^{2}+H^{2}}-\left(\sqrt{D^{2}+(W-L)^{2}+H^{2}}+\right. \\
\left.L_{e f f}\right)+c \Delta \tau<0, \\
L_{e f f}-L>c \Delta \tau .
\end{gathered}
$$

where $c$ is the speed of light.

According to (11), to avoid time overlapping, it is necessary that the difference between the effective length of the transmission line and the separation of the antennas will be larger than the spread of the signal due to both antennas.

As the effective length of the transmission line has to be increased, denser frequency sampling will be demanded to correctly retrieve the AUT pattern. The frequency sampling defined in (7) can be expressed as a function of distance as follows:

$$
\Delta f<\frac{c}{2\left(L_{e f f}+L+c \Delta \tau\right)} .
$$

Finally, as the method relies in the separation of one of the cross-correlation terms in the TD, to be able to filter this term with a minimum error it is necessary to guarantee that there is no time overlap. The field is acquired over a range of frequencies with a bandwidth $B$ so that it can be expressed as:

$$
E(\vec{r}, w)=H_{m}(\vec{r}, w) \Pi\left(\frac{w-w_{c}}{2 \pi B}\right),
$$

being $w_{c}$ the angular frequency of the center of the band and $\Pi(x)$ the pulse function defined as:

$$
\Pi(x)=\left\{\begin{array}{ll}
1 & \text { if }|x| \leq \frac{1}{2} \\
0 & \text { otherwise }
\end{array} .\right.
$$

In the TD, after performing an inverse FT, the field will be spread by means of a convolution with a sinc function modulated with an exponential term, due to the frequency shifted pulse employed in (13):

$$
e(\vec{r}, t)=h_{m}(\vec{r}, t) \otimes B \operatorname{sinc}(B t) e^{j w_{c} t} .
$$

The spread of the sinc function, approximately $1 / B$, could result in the overlap of the TD responses for antennas with narrow bandwidth. For all the aforementioned facts, the method is suitable only for broadband antennas so that the spread of the cross-correlation terms is small enough to allow for a correct filtering of the desired term.

\section{Measurement process}

Prior to the AUT characterization, a calibration process, as in conventional off-axis holography, is needed. This calibration process only has to be done once, and consists on a complete characterization of the reference antenna in amplitude and phase in the same frequency range that will be used during the normal operation of the system.

Once the calibration has been accomplished, the following steps have to be performed:

1) A complete spatial acquisition with a frequency sweep at each point is performed considering only the branch of the AUT. The measured power corresponds to $\left|E_{\text {aut }}(\vec{r}, w)\right|^{2}$.

2) With both antennas connected to the outputs of the directional coupler, the acquisition process is repeated and the measured power corresponds this time to the hologram $H(\vec{r}, w)$.

3) The modified hologram, $H_{m}(\vec{r}, w)$, is computed as in (2) and the phase retrieval algorithm is applied.

4) Steps 1), 2) and 3) are done to retrieve the main polarization of the AUT (the three antennas with the same polarization). In order to acquire the other component of the AUT its polarization needs to be changed, while maintaining the initial position of the probe and the reference antenna. Then steps 1), 2) and 3) have to be repeated.

Finally, once the amplitude and phase of the AUT have been retrieved for both field components, the far-field pattern and the aperture fields of the antenna could be calculated by any suitable method (NF-FF back propagation, source reconstruction technique, etc.).

It is worth noting that the first step, in which the amplitude of the AUT is characterized, is needed to compute the modified hologram, defined as in (2). This extra measurement allows for the removal of the auto-correlation terms of the hologram easing the filtering process. Furthermore, the final retrieved field of the AUT can be composed with this measured amplitude and the retrieved phase, improving error reduction.

Nevertheless, this first step could be omitted, reducing the number of acquisitions to one at the expense of using larger delay lines to increase the separation of the crosscorrelation terms and a denser frequency sampling (see (12)). Error is expected also to be slightly higher for this case as $\left|E_{\text {aut }}(\vec{r}, w)\right|^{2}$ is not measured and, therefore, the retrieved amplitude should be used.

Processing time for the phase retrieval for the proposed method is comparable to the time needed to retrieve the phase with the standard off-axis holography method. As both methods rely on the use of fast Fourier Transforms, the time needed to retrieve the phase is in the order of a few seconds for current computers and, therefore, it is negligible compared to the acquisition time of the data, which can vary from one method to another depending on the measurement system features (single frequency and continuous move acquisitions, frequency sweeps, etc.). 


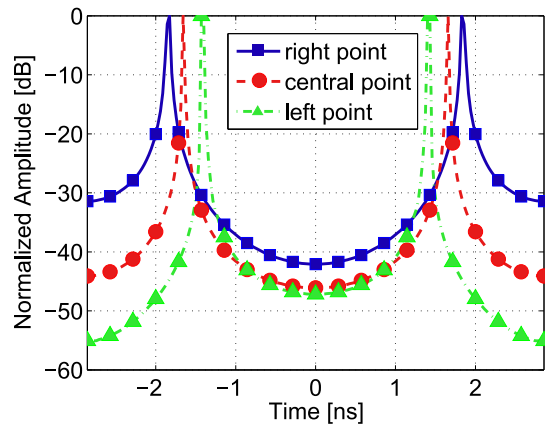

Fig. 4. Modified hologram in the time domain for the dielectric rod antenna. Trace colors are coincident with the study points shown in Fig. 1.

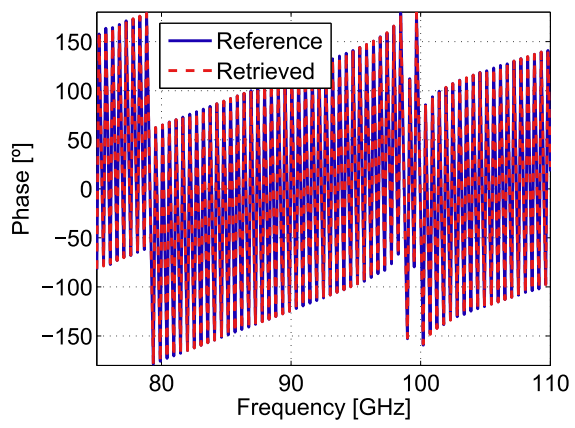

(a)

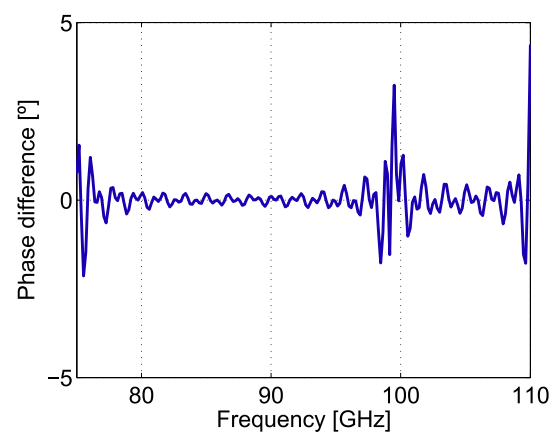

(b)

Fig. 5. Retrieved phase for the worst case in the acquisition plane, i.e. at the left point: (a) Retrieved and simulated phase; (b) Unwrapped difference between simulated and retrieved phase patterns.

\section{NUMERICAL EXAMPLE}

In order to study the capabilities of the phase retrieval algorithm for efficient broadband characterization of antennas, a numerical example for the characterization of a dielectric rod antenna will be shown next.

The rod antenna and the reference antenna (a horn antenna with a radiation pattern modeled as a $\cos ^{q}(\theta)$ with $q=1$ ) have been designed and simulated in the $W$-band, for 201 equally spaced frequency points ranging from $75 \mathrm{GHz}$ to $110 \mathrm{GHz}$, with the computational electromagnetic software FEKO [28]. The frequency sampling rate has been set so that the requirement of (12) is thoroughly fulfilled.

Separation between the antennas is $L=15 \mathrm{~cm}$ and the acquisition plane, defined at $D=50 \mathrm{~cm}$ from the AUT antenna, is a $50 \mathrm{~cm} \times 50 \mathrm{~cm}$ square with a sampling of $6 \mathrm{~mm}$ for both dimensions, being all the distances referred to the

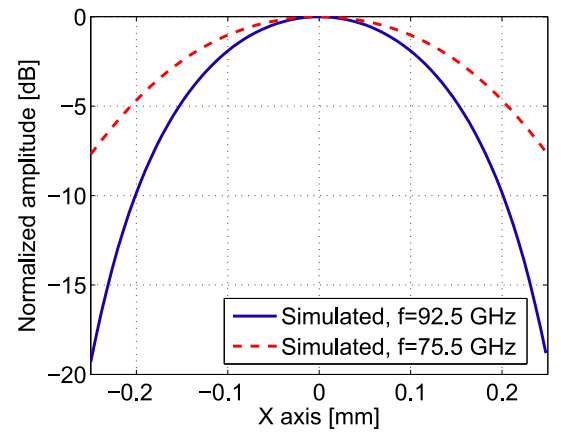

(a)

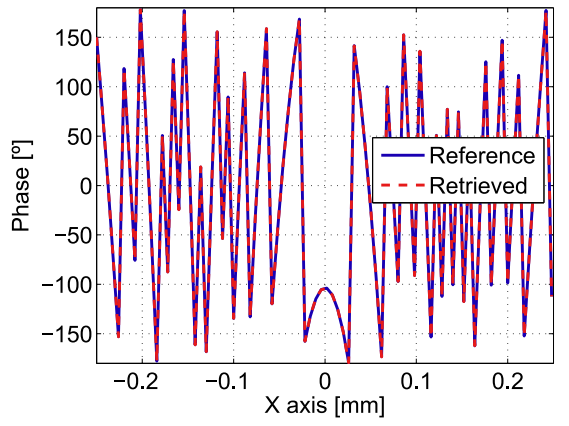

(b)

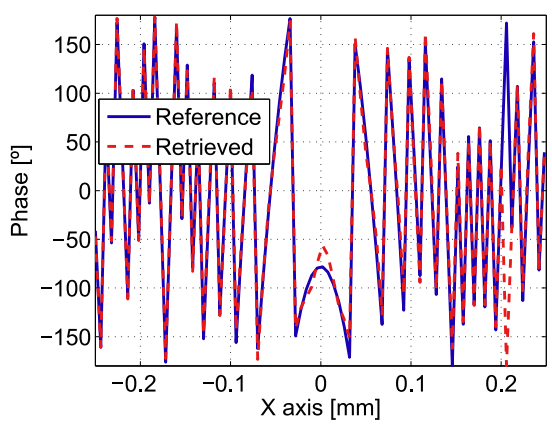

(c)

Fig. 6. Main cut of the H-plane for the dielectric rod antenna in the $W$ band. Comparison between retrieved and reference fields: (a) Normalized simulated amplitude for $92.5 \mathrm{GHz}$ and $75.5 \mathrm{GHz}$; (b) Reference (simulated) and retrieved phase for $92.5 \mathrm{GHz}$; (c) Reference (simulated) and retrieved phase for $75.5 \mathrm{GHz}$.

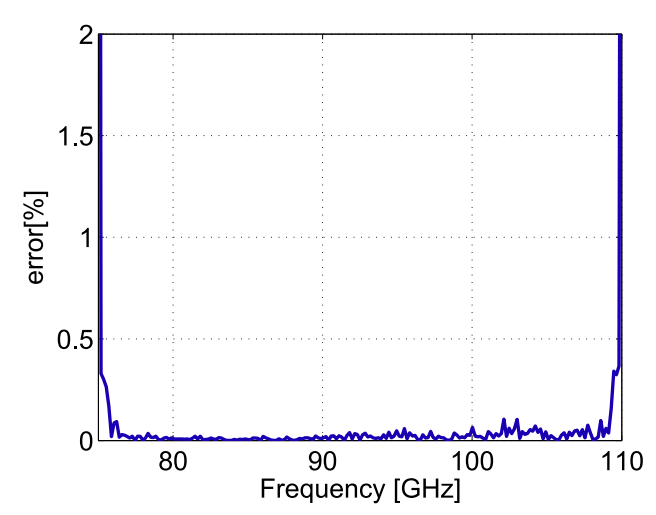

Fig. 7. Error of the phase retrieval algorithm for the dielectric rod antenna in the $W$-band. 
center of the aperture planes of the antennas. The reference antenna has a tilt of $\alpha=73^{\circ}$ so it is pointing to the center of the acquisition plane and is connected to one of the outputs of the directional coupler by means of a transmission line that introduces a delay of $0.8 \mathrm{~ns}$ which corresponds to a $L_{e f f}$ of $144 \mathrm{~mm}$ if 0.6 is considered as the velocity factor (see Fig. 1). It is important to note that, for alleviating simulation time, sampling is larger than $\lambda / 2$ for all the frequency band, and therefore, the retrieved data could not be used to perform a conventional aliasing free FF transformation.

Results for the E-plane ( $\mathrm{y}=0$ cut) copolar component are shown to illustrate the setup and the phase retrieval method capabilities.

Fig. 4 shows the modified hologram in the time domain as defined in (3) for three different points in the acquisition plane (shown in Fig. 1 with the same color and marker code). Although the position of those cross-correlation terms varies depending on the time the signals need to travel from the AUT and the reference antenna to the probe antenna, there is no overlap between the cross-correlation terms, and the desired term can be filtered for all the acquisition points with a rectangular window defined from $-3 \mathrm{~ns}$ to $0 \mathrm{~ns}$.

Fig. 5 a shows the retrieved phase for all the frequencies for the worst case of the spatial acquisition: as previously mentioned, this case corresponds to an acquisition point on the left side of the acquisition plane, on the reference antenna side (triangular marker in Fig. 1); the points on this side are closer to the reference antenna than to the AUT, so the second expression in (6) is minimized (dash-dotted hologram in Fig. 4). As it can be seen in Fig. 5 b for this case the phase is retrieved almost without error, being the maximum difference between the reference phase and the retrieved one of $3^{\circ}$ in the central part of the band.

Once the phase is retrieved for all the acquisition points, the $\mathrm{NF}$ of the AUT in the acquisition plane can be obtained for each frequency. The measured amplitude and retrieved phase for the frequencies of $75.5 \mathrm{GHz}$ and $92.5 \mathrm{GHz}$ are shown in Fig. 6. The retrieved phase is compared to the phase obtained from simulations.

The retrieved phase of the AUT for the frequency of $92.5 \mathrm{GHz}$, central frequency of the band, is in very good agreement with the reference field obtained from the simulations with amplitude and phase data (error of $0.002 \%$ ). For $75.5 \mathrm{GHz}$, in the lower part of the band, the retrieved phase has some discrepancies (error of $0.3 \%$ ).

To quantify the quality of the phase retrieval method, the following error is calculated for all the frequencies:

$$
\text { error }[\%]=100 \frac{\left\|E_{\text {sim }}-E_{\text {ret }}\right\|_{2}}{\left\|E_{\text {sim }}\right\|_{2}},
$$

where $\|\cdot\|_{2}$ is the Euclidean norm, $E_{\text {sim }}$ is the field of the AUT obtained from simulations with phase information, and $E_{r e t}$ is the retrieved field of the AUT. The graphical representation of the error is shown in Fig. 7. Due to the filtering in the $\mathrm{TD}$, the error is higher in the extremes of the frequency band [29], and therefore, it is advisable to discard the results in those extremes. The range of frequencies contaminated by this error is minimum in the case of using a

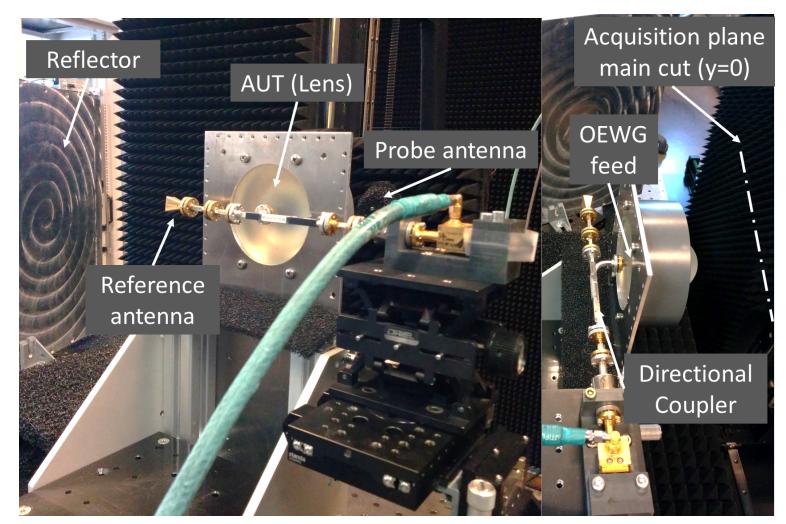

(a)

(b)

Fig. 8. Setup for the $W$-band antenna measurement: (a) side view; (b) top view.

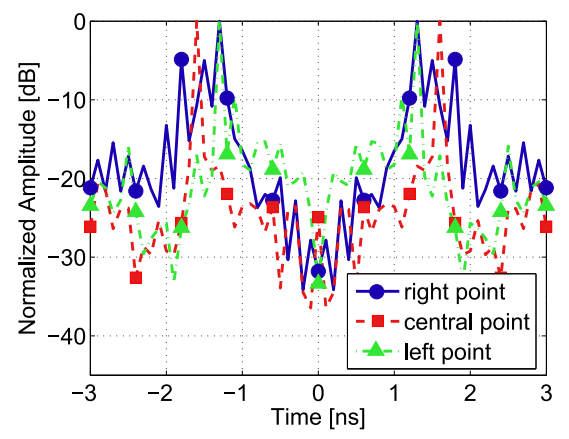

Fig. 9. Modified hologram in the time domain for the lens antenna. Trace colors are coincident with the study points shown in Fig. 1.

rectangular window, as the one used in this work. However, it could be of interest to use other windows to reduce the mean error [29]. For this simulation example, mean error of the retrieval algorithm is $0.14 \%$.

\section{Measurement Example}

Two measurement examples with different types of antennas with different directivities and working frequency bands are presented to completely validate the method and the phaseretrieval algorithm. Slight modifications of the basic setup (see Fig. 1) such as the use of reflectors or amplifiers are introduced respectively in each of the presented setups.

\section{A. W-band lens antenna measurement}

A lens antenna was characterized in the $W$-band. The measurements were performed with the MilliLab PNF scanner described in [8]. The main components of the setup and the layout can be seen in Fig. 8.

The lens antenna is a $64 \mathrm{~mm}$ circular lens with a $1 \mathrm{~cm}$ length WR-10 OEWG feed, and a $20 \mathrm{~dB}$ gain horn is used as a reference antenna, both with horizontal polarization. Separation between the center of the aperture of the antennas is $105 \mathrm{~mm}$ yielding an effective length $\left(L_{e f f}\right)$ of $63 \mathrm{~mm}$ considering a velocity factor of $0.6 c$. Mirror reflection of the reference signal is used in order to increase the path of the reference branch signal. The reflector, a planar metallic plate of 


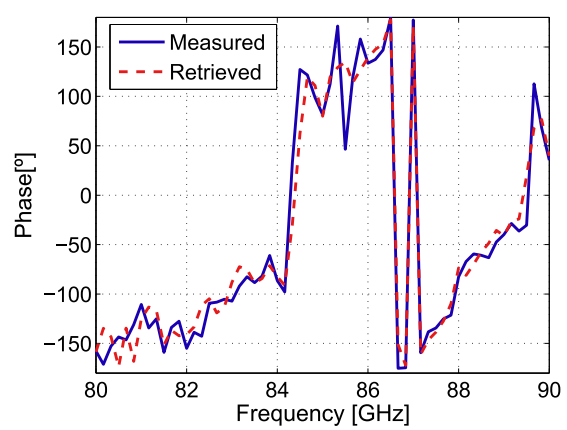

(a)

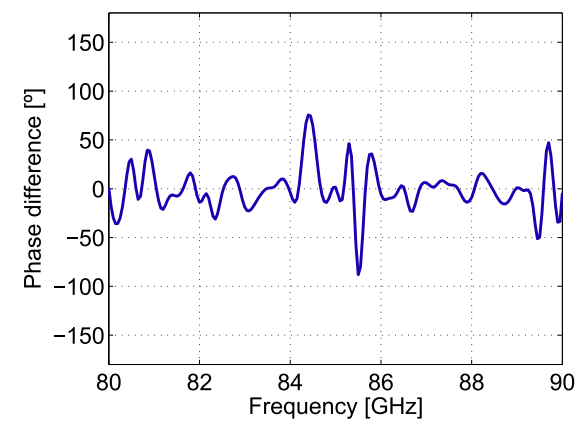

(b)

Fig. 10. Retrieved phase for the worst case in the acquisition plane, i.e. at the left point: (a) Retrieved and measured phase; (b) Difference between retreieved and measured phase patterns.

$210 \mathrm{~mm} \times 300 \mathrm{~mm}$, is placed at $220 \mathrm{~mm}$ of the aperture plane of the reference antenna with a tilt of $60^{\circ}$ so that the acquisition plane, at $150 \mathrm{~mm}$ of the aperture of the AUT, is illuminated with an almost constant amplitude.

The field acquisitions are performed for a cut in the $x$ axis of $200 \mathrm{~mm}$ with spatial sampling of $\lambda_{m} / 2$, being $\lambda_{m}$ the wavelength at maximum acquisition frequency $(90 \mathrm{GHz})$. The measurement system does not allow for simultaneous multi-frequency acquisitions, therefore, 61 different spatial acquisitions for equally spaced frequencies between $80 \mathrm{GHz}$ and $90 \mathrm{GHz}$ have been done, guaranteeing the requirements in (12) are fulfilled.

With the purpose of quantifying the accuracy of the phase retrieval algorithm, AUT field amplitude and phase data acquisition is performed thus a comparison between the retrieved and the measured phase can be done.

As only a fraction of the bandwidth of the AUT is being characterized, the effect of the sinc convolution defined in (15) will introduce a spread in the cross-correlation terms of the spectrum complicating the filtering of the desired term. Nevertheless the frequency sampling is enough to retrieve the phase of the AUT with an acceptable error level.

As in the numerical example, the results are presented for the E-plane ( $\mathrm{y}=0$ cut).

Fig. 9 shows the modified hologram for three different points in the acquisition plane, using the color code indicated in Fig. 1. As it is expected, separation between crosscorrelation terms decreases as the probe antenna is closer to the reference antenna, although for this case, the crosscorrelation terms associated to the point in the right side of the acquisition plane (opposite to the reference antenna side) are spread, having its maximum in the same position than the terms corresponding to the left side of the acquisition plane. This might be due to some reflections of the reference signal with the holding structure for the AUT and also due to the poor resolution of the spectrum caused by the sparse frequency sampling.

A comparison between the retrieved and the measured phase for all the frequencies corresponding to the worst case scenario, probe antenna closer to the reference antenna, is shown in Fig. 10a. There are some discrepancies that can be clearly seen in Fig. 10b, showing the difference pattern. The differences are noticeable in the central part of the band, wherein the measured phase have some abrupt variations that are not present in the retrieved phase, probably due to the low density of the frequency sampling and because the signal level is lower in this section of the band.

Fig. 11 shows the measured amplitude and the retrieved phase of the AUT for two different frequencies, $87 \mathrm{GHz}$ and $83.5 \mathrm{GHz}$. Both results of retrieved phase are in very good agreement with the measurements, being the error of the phase retrieval of $2.73 \%$ and $3.24 \%$ respectively for each frequency.

Finally, Fig. 12 shows the error of the phase retrieval for each of the frequencies of the band as defined in (16); mean error is $2.40 \%$. Excluding the extremes of the frequency band, wherein the error rises due to the TD filtering, the highest level of error occurs at $80.8 \mathrm{GHz}$ due to the different the level of the signals from the AUT and reference antenna in the acquisition plane.

This could be corrected by employing a variable attenuator to balance the signal level in both branches increasing the dynamic range of the system [21].

\section{B. $K_{a}$-band pyramidal horn antenna measurement}

A $20-\mathrm{dB}$ gain pyramidal horn antenna is characterized in the complete $K_{a}$-band from $26.5 \mathrm{GHz}$ to $40 \mathrm{GHz}$ in this measurement example. The measurements were done with the TSC-Uniovi PNF scanner [13]. The components and the layout can be seen in Fig. 13.

The reference antenna, a small horn, vertically polarized, is placed at $200 \mathrm{~mm}$ of the AUT (distances are measured from the center of the aperture planes of the antennas) and with a tilt of $52.5^{\circ}$ so that its maximum field is outside the acquisition plane and the illumination of the acquisition plane is as uniform as possible. The reference antenna is connected to the directional coupler by means of an $80 \mathrm{~cm}$ coaxial cable whose effective length can be approximated by $48 \mathrm{~cm}$ considering a velocity factor of 0.6 . The acquisition plane, at $260 \mathrm{~mm}$ of the AUT aperture is a $300 \mathrm{~mm} \times 300 \mathrm{~mm}$ square. Spatial sampling is $3.7 \mathrm{~mm}$, that is $\lambda / 2$ at $40 \mathrm{GHz}$. For each spatial point, a frequency sweep of 401 points is performed, to thoroughly fulfill the requirements in (12). An amplifier is included in the AUT branch to balance the signal level of the AUT and the reference signal.

Results are shown for the H-plane $(y=0)$ cut. The modified hologram in the TD can be seen in Fig. 14 for the three different points in the main cut of the acquisition plane 
This is the author's version of an article that has been published in this journal. Changes were made to this version by the publisher prior to publication.

The final version of record is available at http://dx.doi.org/10.1109/TAP.2015.2511789

IEEE TRANSACTIONS ON ANTENNAS AND PROPAGATION, VOL. X, NO. X, MONTH 2015

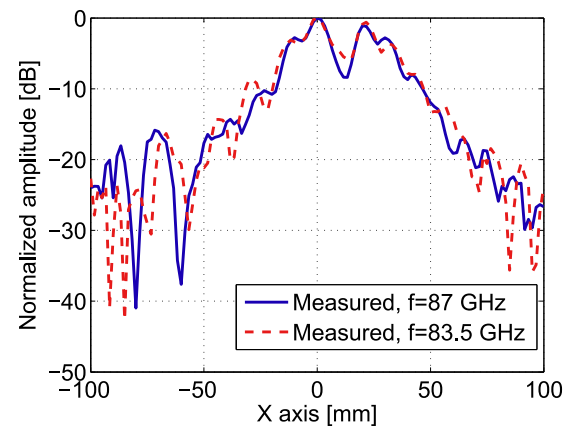

(a)

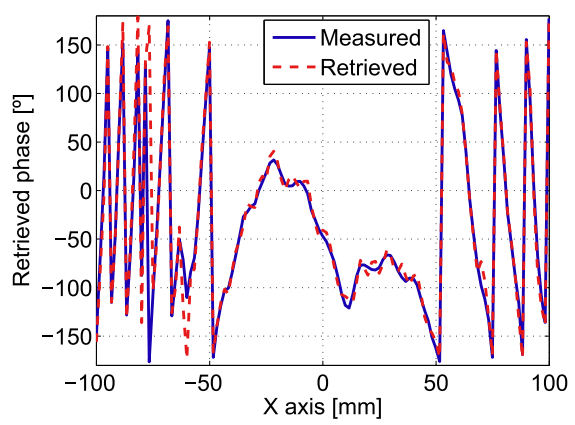

(b)

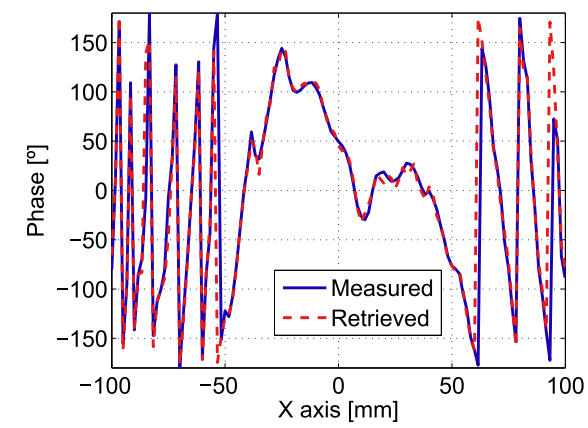

(c)

Fig. 11. Main cut of the $\mathrm{H}$-plane for the lens antenna in the $W$-band Comparison between retrieved and measured fields: (a) Normalized measured amplitude for $87 \mathrm{GHz}$ and $83.5 \mathrm{GHz}$; (b) Measured and retrieved phase for $87 \mathrm{GHz}$; (c) Measured and retrieved phase for $83.5 \mathrm{GHz}$.

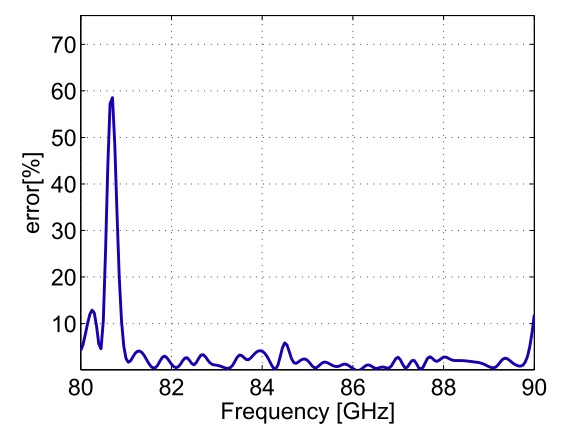

Fig. 12. Error of the phase retrieval algorithm for the lens antenna in the $W$-band.

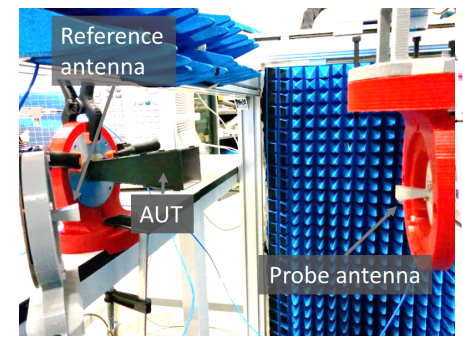

(a)

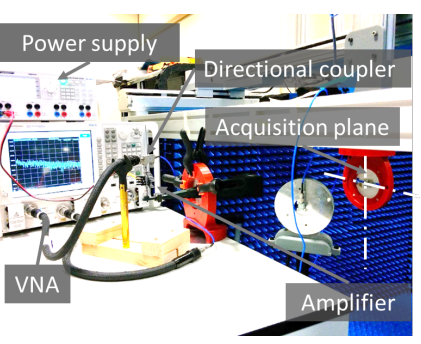

(b)
Fig. 13. Setup for the $K_{a}$-band antenna measurement: (a) position of the antennas; (b) complete setup.

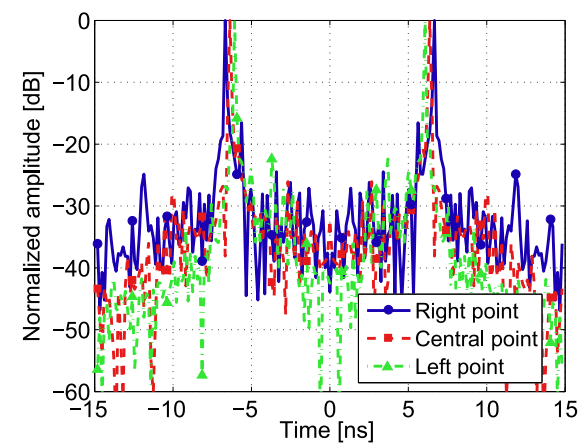

Fig. 14. Modified hologram in the time domain for the pyramidal horn antenna. Trace colors are coincident with the study points shown in Fig. 1.

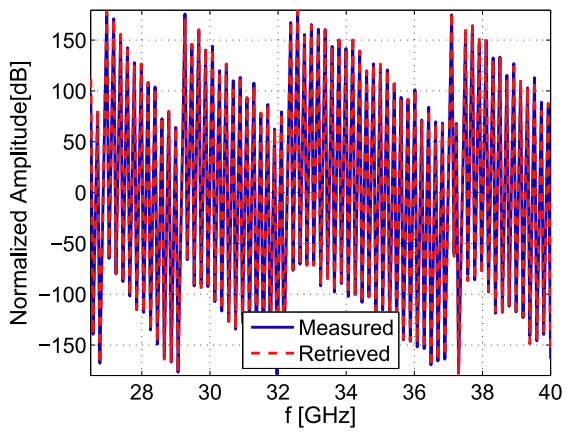

(a)

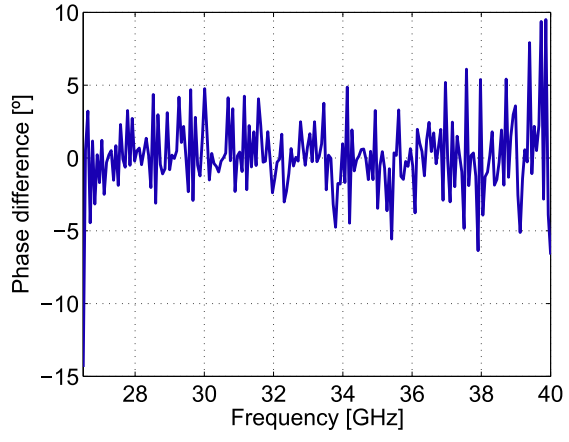

(b)

Fig. 15. Retrieved phase for the worst case in the acquisition plane, i.e. at the left point: (a) Retrieved and measured phase; (b) Unwrapped difference between retrieved and measured phase patterns. 


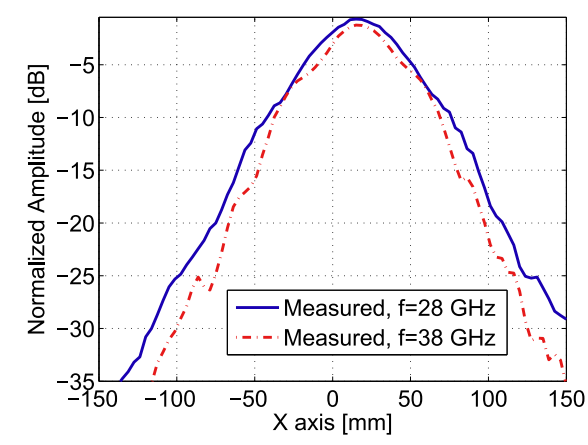

(a)

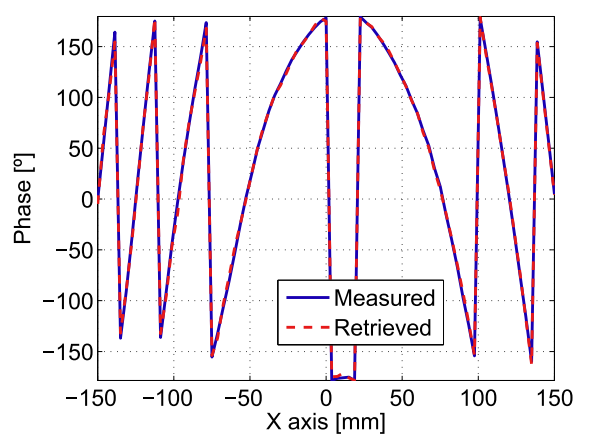

(b)

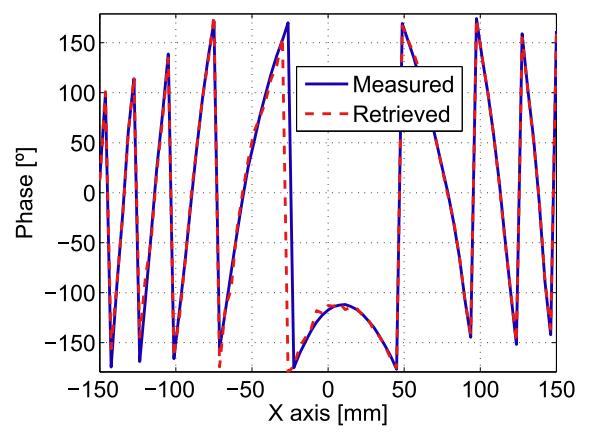

(c)

Fig. 16. Main cut of the E-plane for the pyramidal horn antenna in the $K_{a}$-band. Comparison between retrieved and measured fields: (a) Normalized measured amplitude for $28 \mathrm{GHz}$ and $38 \mathrm{GHz}$; (b) Measured and retrieved phase for $28 \mathrm{GHz}$; (c) Measured and retrieved phase for $38 \mathrm{GHz}$.

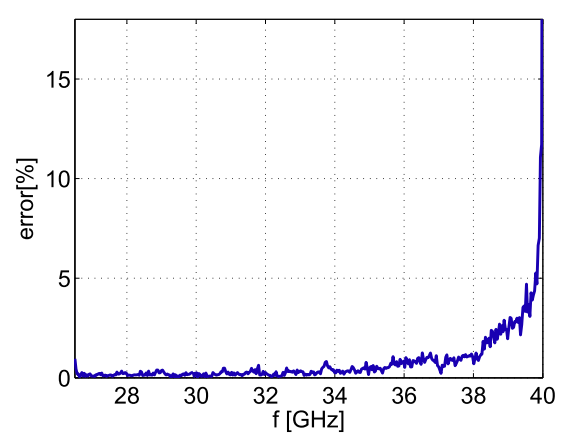

Fig. 17. Error of the phase retrieval algorithm for the horn antenna in the $K_{a}$-band. highlighted in Fig. 1. As it can be clearly seen, all of the terms of the modified hologram can be easily filtered. A rectangular window $8.8 \mathrm{~ns}$ wide, centered in the maximum of the corresponding cross-correlation term in the negative side of the spectrum, will be used to filter the hologram in each spatial acquisition point.

The retrieved phase for the worst case (point with the triangular marker in Fig.1) is shown in Fig. $15 \mathrm{a}$ and is in very good agreement with the measured phase. The difference pattern between the measured and the retrieved phase is depicted in Fig. 15 b. For most of the points, the error is less than $6^{\circ}$.

As in the previous examples, the final retrieved field is composed by the measured amplitude and the retrieved phase, and for this case it is depicted for $28 \mathrm{GHz}$ and $38 \mathrm{GHz}$ in Fig. 16. The retrieved fields are in perfect accordance with the measured ones, being the error $0.14 \%$ and $1.07 \%$ respectively for 28 and $38 \mathrm{GHz}$.

The representation of the error for the complete frequency band, as defined in (16) is shown in Fig. 17 being the mean error value $0.83 \%$. The mean error from $37 \mathrm{GHz}$ to $40 \mathrm{GHz}$ is much more higher $(3.68 \%)$ than for the rest of the band. Apart from the error introduced in the frequency band edges due to the TD filtering, the level of the reference branch decays in that upper part of the band causing a reduction of the dynamic range of the system, which explains the increase of the error.

If the hologram is processed without subtracting the amplitude of the AUT, which means that only one acquisition is needed for each field component characterization, but disabling the possibility of auto-correlation terms removal, the mean error of the phase retrieval increases from $0.83 \%$ to $4.12 \%$.

The FF pattern of the data obtained with the proposed phase retrieval method is computed through the standard plane-rectangular NF-FF transformation [14] and compared to the FF pattern obtained from a set of NF data with direct phase acquisition. The uncertainty for the peak-level and the secondary lobe level (SLL) for both FF patterns is calculated following the procedure described in [30] in order to quantify the error introduced in the FF pattern when using phaseless acquisitions.

The procedure employs the root mean square (RMS) value of the equivalent stray signal (ESS) obtained as the difference of both FF patterns (the one obtained from amplitude and phase NF data and the one obtained from the phaseless acquisition) to calculate the uncertainty. The uncertainty values together with the RMS value of the ESS, are shown in Table I for the frequencies of $28 \mathrm{GHz}$ and $38 \mathrm{GHz}$.

It is worth noting that for the frequency of $38 \mathrm{GHz}$, the phase retrieval error is quite high (see Fig. 17) and therefore, higher values of uncertainty are expected.

For this setup, the angular valid margin of the NF-FF transformation is $25^{\circ}$ [14]. The secondary lobes are close to the previous defined limit so the uncertainty values have to be read carefully for this example. Despite of that, the uncertainty parameter for the frequency of $28 \mathrm{GHz}$ is low and the one obtained for the frequency of $38 \mathrm{GHz}$ is acceptable considering the error of the phase retrieval for this frequency 


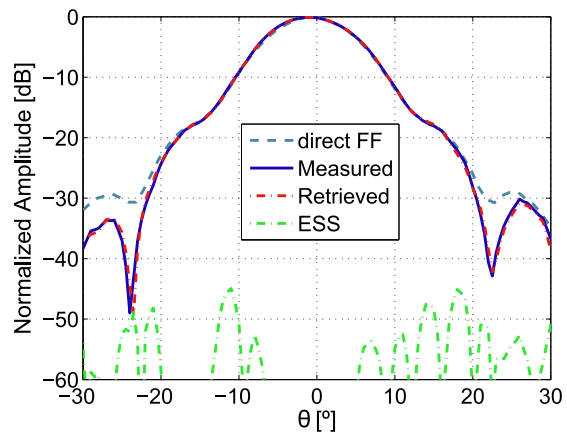

(a)

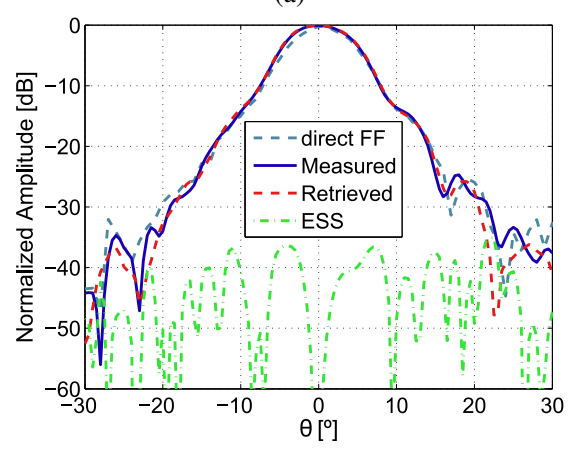

(b)

Fig. 18. Comparison of the FF patterns for the horn antenna in the $k_{a}$-band for the case of using direct FF acquisition, a set of NF amplitude-only data and the phase retrieval method and a complex NF acquisition with amplitude and phase. The ESS is calculated for both cases: (a) $E_{\theta}$ cut for $\phi=0$ at $28 \mathrm{GHz}$; (b) $E_{\theta}$ cut for $\phi=0$ at $38 \mathrm{GHz}$.

TABLE I

UNCERTAINTIES IN THE FF PATTERN CALCULATION FOR THE MAIN PARAMETERS OF THE RADIATION DIAGRAMS (THE SLL UNCERTAINTY IS CALCULATED CONSIDERING A SLL OF $-33.5 \mathrm{~dB}$ ).

\begin{tabular}{cccc}
\hline \hline frequency & ESS & Peaklevel & SLL \\
{$[G H z]$} & $R M S[d B]$ & uncertainty $[d B]$ & uncertainty $[d B]$ \\
\hline 28 & -61.1509 & 0.0076 & 0.3527 \\
38 & -47.5861 & 0.0362 & 1.5601 \\
\hline \hline
\end{tabular}

is quite high. The uncertainty parameters regarding the peak level are extremely low for both frequencies.

The $E_{\theta}$ cut for $\phi=0$ of the FF patterns and the ESS are shown in Fig. 18. Finally, a direct FF acquisition over an spherical range is included for comparison purposes. Differences with respect to planar range with NF-FF transformation are mainly due to the planar range truncation error.

\section{CONCLUSION}

A new efficient method for broadband phaseless characterization of antennas from just two surface acquisitions is presented. The method is a modification of the conventional off-axis holography scheme and is presented for planar acquisitions, although extrapolation to other geometries is straightforward.

The phase retrieval is done independently point-by-point in the acquisition plane, after the field is acquired for a set of frequencies, by calculating and filtering the hologram in the time domain. Therefore, the proposed technique does not depend on the acquisition geometry nor on the accuracy of the positioners, making the method suitable to work at high frequency bands.

The method does not require increasing the spatial bandwidth to reduce spectral overlapping, as it happens in conventional off-axis holography methods, resulting in less acquisition points. In contrast, time responses overlap of the crosscorrelation terms of the hologram can be easily controlled by introducing enough delay in the reference antenna branch or, alternatively, in the AUT branch.

The error of the phase retrieval and the dynamic range of the system are correlated to the power difference between the AUT and the reference branches; thus, the use of a variable attenuator to help leveling those branches is recommended.

Main drawback is that all the involved components, mainly the AUT, must be broadband. If the antenna is narrowband, the computed time responses will be spread (convoluted with a sinc of width $1 / B$ ) and it could result in the overlap of the time domain responses. Also the complete characterization of the reference antenna in the acquisition plane is needed.

The method has been illustrated with several examples in the microwave and millimeter-wave bands. Very good results are obtained for all the studied cases.

\section{REFERENCES}

[1] Y. Huang, K. Chan, and B. Cheeseman, "Review of broadband antenna measurements," in 1st European Conf. on Antennas and Propag.. EuCAP 2006, Nov. 2006, pp. 1-4.

[2] R. de Jongh, M. Hajian, and L. Ligthart, "Time domain antenna measurements: implementation and verification of a novel technique," in 27th European Microwave Conf., vol. 1, Sep. 1997, pp. 470-475.

[3] Y. Huang, M. Bury, Y. Yashchyshyn, A. Yarovoy, R. De Jongh, S. Maqbool, and Y. Lu, "Time-domain measurements of broadband antennas," in 2nd European Conf. on Antennas and Propag.. EuCAP 2007, Nov. 2007, pp. $1-4$.

[4] A. Kalinin, "Anechoic chamber wideband antenna measurements," IEEE Aerosp. Electron. Syst. Mag., vol. 21, no. 1, pp. 21-24, Jan. 2006.

[5] T. Hansen and A. Yaghjian, "Planar near-field scanning in the time domain. Formulation," IEEE Trans. Antennas Propag., vol. 42, no. 9, pp. 1280-1291, Sep. 1994.

[6] _ - "Formulation of probe-corrected planar near-field scanning in the time domain," IEEE Trans. Antennas Propag., vol. 43, no. 6, pp. 569584, Jun. 1995.

[7] G. Junkin, T. Huang, and J. Bennett, "Holographic testing of terahertz antennas," IEEE Trans. Antennas Propag., vol. 48, no. 3, pp. 409-417, Mar. 2000.

[8] A. Räisänen, J. Ala-Laurinaho, A. Karttunen, J. Mallat, A. Tamminen, and M. Vaaja, "Measurements of high-gain antennas at $\mathrm{THz}$ frequencies," in 4th European Conf. on Antennas and Propag. (EuCAP), April 2010, pp. 1-3.

[9] A. V. Raisanen and J. Ala-Laurinaho, "Holographic principles in antenna metrology at millimeter and submillimeter wavelengths," in Antennas and Propagation (EuCAP), 2015 9th European Conference on, April 2015, pp. 1-2.

[10] A. Newell, "Error analysis techniques for planar near-field measurements," IEEE Transactions on Antennas and Propagation, vol. 36, no. 6, pp. 754-768, Jun. 1988.

[11] Y. Rahmat-Samii, L. Williams, and R. Yaccarino, "The UCLA bi-polar planar-near-field antenna-measurement and diagnostics range," IEEE Antennas Propag. Mag., vol. 37, no. 6, pp. 16-35, Dec. 1995.

[12] T. Brockett and Y. Rahmat-Samii, "A novel portable bipolar nearfield measurement system for millimeter-wave antennas: construction, development, and verification," IEEE Antennas Propag. Mag., vol. 50, no. 5, pp. 121-130, Oct. 2008.

[13] A. Arboleya, Y. Álvarez, and F. Las-Heras, "Millimeter and submillimeter planar measurement setup," in 2013 IEEE Antennas and Propag. Soc. Int. Symp. (APSURSI), Jul. 2013, pp. 1-2.

[14] A. Yaghjian, "An overview of near-field antenna measurements," IEEE Trans. Antennas Propag., vol. 34, no. 1, pp. 30-45, Jan. 1986. 
[11] Y. Rahmat-Samii, L. Williams, and R. Yaccarino, "The UCLA bi-polar planar-near-field antenna-measurement and diagnostics range," IEEE Antennas Propag. Mag., vol. 37, no. 6, pp. 16-35, Dec. 1995.

[12] T. Brockett and Y. Rahmat-Samii, "A novel portable bipolar nearfield measurement system for millimeter-wave antennas: construction, development, and verification," IEEE Antennas Propag. Mag., vol. 50, no. 5, pp. 121-130, Oct. 2008.

[13] A. Arboleya, Y. Álvarez, and F. Las-Heras, "Millimeter and submillimeter planar measurement setup," in 2013 IEEE Antennas and Propag. Soc. Int. Symp. (APSURSI), Jul. 2013, pp. 1-2.

[14] A. Yaghjian, "An overview of near-field antenna measurements," IEEE Trans. Antennas Propag., vol. 34, no. 1, pp. 30-45, Jan. 1986.

[15] J. Wang, "An examination of the theory and practices of planar nearfield measurement," IEEE Trans. Antennas Propag., vol. 36, no. 6, pp. 746-753, Jun. 1988.

[16] S. Razavi and Y. Rahmat-Samii, "A new look at phaseless planar near-field measurements: limitations, simulations, measurements, and a hybrid solution," IEEE Antennas Propag. Mag., vol. 49, no. 2, pp. 170178, Apr. 2007.

[17] F. Las-Heras and T. Sarkar, "A direct optimization approach for source reconstruction and NF-FF transformation using amplitude-only data," IEEE Trans. Antennas Propag., vol. 50, no. 4, pp. 500-510, Apr. 2002.

[18] D. Smith, M. Leach, M. Elsdon, and S. Foti, "Indirect holographic techniques for determining antenna radiation characteristics and imaging aperture fields," IEEE Antennas Propag. Mag., vol. 49, no. 1, pp. 54-67, Feb. 2007

[19] V. Schejbal, V. Kovarik, and D. Cermak, "Synthesized-reference-wave holography for determining antenna radiation characteristics," IEEE Antennas Propag. Mag., vol. 50, no. 5, pp. 71-83, Oct. 2008.

[20] J. Laviada and F. Las-Heras, "Phaseless antenna measurement on nonredundant sample points via Leith-Upatnieks holography," IEEE Trans. Antennas Propag., vol. 61, no. 8, pp. 4036-4044, Aug. 2013.

[21] J. Laviada Martínez, A. Arboleya-Arboleya, Y. Álvarez-López, C. García-González, and F. Las-Heras, "Phaseless antenna diagnostics based on off-axis holography with synthetic reference wave," IEEE Antennas Wireless Propag. Lett., vol. 13, pp. 43-46, 2014.

[22] V. Schejbal, J. Pidanic, V. Kovarik, and D. Cermak, "Accuracy analyses of synthesized-reference-wave holography for determining antenna radiation characteristics," IEEE Antennas Propag. Mag., vol. 50, no. 6 , pp. 89-98, Dec. 2008.

[23] D. Smith, S. Skobelev, and M. Leach, "A modified holographic technique for antenna measurements," in 8th Int. Symp. on Antennas, Propag. and EM Theory. ISAPE 2008, Nov. 2008, pp. 46-49.

[24] D. Gabor, "Microscopy by reconstructed wave-fronts," Proc. of the Royal Soc. of London A: Math., Physical and Eng. Sci., vol. 197, no. 1051, pp. 454-487, Jul. 1949.

[25] E. N. Leith and J. Upatnieks, "Reconstructed wavefronts and communication theory," Journal of the Optical Soc. of America, vol. 52, no. 10, pp. 1123-1128, Oct. 1962.

[26] S. Costanzo and G. Di Massa, "Wideband phase retrieval technique from amplitude-only near-field data," Radioengineering, vol. 17, no. 4, pp. 8 12, Feb. 2008.

[27] S. Costanzo, G. Di Massa, and D. Migliore, "A novel hybrid approach for far-field characterization from near-field amplitude-only measurements on arbitrary scanning surfaces," IEEE Trans. Antennas Propag., vol. 53 , no. 6, pp. 1866-1874, Jun. 2005.

[28] "FEKO Suite 6.2 features." [Online]. Available: https://www.feko.info/about-us/News/feko-suite-6.2-feature-updateavailable .

[29] J. Laviada, A. Arboleya-Arboleya, Y. Álvarez-López, C. GarcíaGonzález, and F. Las-Heras, "Phaseless synthetic aperture radar with efficient sampling for broadband near-field imaging: theory and validation," IEEE Trans. Antennas Propag., vol. 2, no. 2, pp. 573-584, Feb. 2015.

[30] A. Newell and G. Hindman, "Antenna pattern comparison using pattern subtraction and statistical analysis," in 5th European Conf. on Antennas and Propag. (EuCAP), Apr. 2011, pp. 2684-2687.

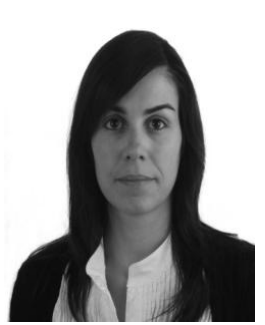

Ana Arboleya received de M.Sc. degree in telecommunication engineering from the University of Oviedo, Spain, in 2009, where she is currently working toward the Ph.D. degree. Since 2008, she has been working as a Research Assistant within the Signal Theory and Communications research group, TSC-UNIOVI, at the Department of Electrical Engineering in the University of Oviedo.

She was a Visiting Scholar in 2014 and 2015 in the Department of Radio Science and Engineering and MilliLab, in Aalto University, Finland.

Her major research interests comprise antenna diagnostics and measurement systems and techniques and high frequency imaging techniques and applications.

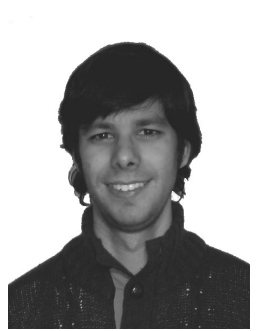

Jaime Laviada was born in Gijn, Spain. He received the M.S. degree in telecommunication engineering and the Ph.D. degree from the Universidad de Oviedo, Spain, in 2005 and 2010, respectively.

In 2006, he joined the research group Signal Theory and Communications of the Universidad de Oviedo, where he has been involved in multiple national and European projects as well as contracts with several companies. He was a Visiting Scholar in the Electromagnetics and Communications Lab, Pennsylvania State University, during 2007 and 2008. In 2015, he moved to the Antennas Group of the Universidad Pública de Navarra with a national postdoctoral fellowship collaborating in several applied research projects. Finally, he moved back to the Universidad de Oviedo where he currently holds a position as assistant professor.

His main research interests are in numerical techniques applied to imaging, antenna measurements, method of moments, and antenna pattern synthesis.

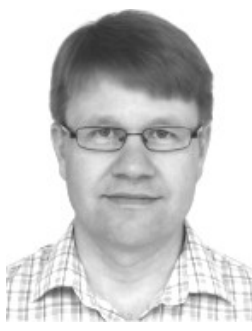

Juha Ala-Laurinaho was born in Parkano, Finland, in 1969. He received the Diploma Engineer (M.Sc.) degree in mathematics and D.Sc. (Tech.) degree in electrical engineering from TKK Helsinki University of Technology, Espoo, Finland, in 1995 and 2001, respectively. He has been with the TKK serving in the Radio Laboratory in 1995-2007 and from 2008 in the Department of Radio Science and Engineering, which is currently a part of the Aalto University School of Electrical Engineering. During 1995, he worked as a Research Assistant and, since 1996, he has been a Research Associate and currently he works as a Staff Scientist. Dr. Ala-Laurinaho has been a Project Manager in many millimeter wave technology related projects. His current research interests are the antennas and antenna measurement techniques for millimeter and submillimeter waves, and the millimeter wave imaging.

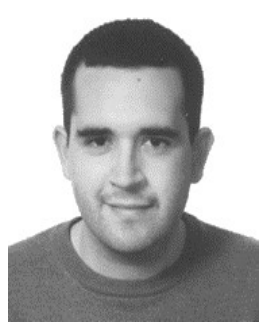

Yuri lvarez (S06 M09 SM15) was born in Langreo, Spain, in 1983. He received the M.S. and Ph.D. degrees in telecommunication engineering from the University of Oviedo, Gijón, Spain, in 2006 and 2009 , respectively. He was a Visiting Scholar at the Department of Electrical Engineering and Computer Science at Syracuse University, Syracuse, NY, USA, in 2006 and 2008, respectively; a Visiting Postdoc at the Gordon Center for Subsurface Sensing and Imaging Systems (CenSSIS)ALERT Center of Excellence, Northeastern University, Boston, MA, USA, from 2011 to 2014; and a Visiting Postdoc at ELEDIA Research Center (Trento, Italy), in 2015. He is currently an Assistant Professor at the Signal Theory and Communications of the University of Oviedo, Gijón, Spain. His interests and research studies have been focused on antenna diagnostics, antenna measurement techniques, RF techniques for indoor location, inverse scattering and imaging techniques, and phaseless methods for antenna diagnostics and imaging. Dr. Alvarez received the 2011 Regional and National Awards to the Best Ph.D. Thesis on Telecommunication Engineering (category: security and defense). 


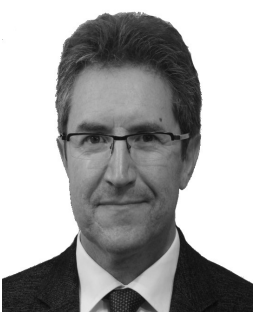

Fernando Las-Heras (M'86, SM'08) received the M.S. in 1987 and the Ph.D. in 1990, both in Telecommunication Engineering, from the Technical University of Madrid (UPM). From 1988 to 1990 he was a National Graduate Research Fellow. From 1991 to 2000 he held a position of Associate Professor at the Department of Signal, Systems and Radiocommunications of the UPM. From December 2003 he holds a Full-Professor position at the University of Oviedo where he was the Vice-dean for Telecommunication Engineering at the Technical School of Engineering at Gijón from 2004 to 2008. As of 2001 he heads the research group Signal Theory and Communications TSC-UNIOVI at the Dept. of Electrical Engineering of the University of Oviedo.

He was a Visiting Lecturer at the National University of Engineering in Peru in 1996, a Visiting Researcher at Syracuse University, New York, in 2000, and a short term Visiting Lecturer at ESIGELEC in France from 2005 to 2011. From 2005 he holds the Telefónica Chair ICTs and Smartcities at the University of Oviedo and from 2010 he is a member of the Science, Technology and Innovation Council of Asturias, and member of the board of directors of the IEEE Spain Section.

He has authored over 300 articles published in academic journals and proceedings of international conferences, mainly in the areas of antenna design and the inverse electromagnetic problem with applications in diagnostic measurement and synthesis of antennas, phaseless techniques, propagation, and microwave to $\mathrm{THz}$ imaging and localization, as well as in engineering education

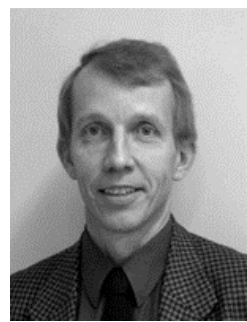

Antti V. Räisänen (F'94) received the D.Sc.(Tech.) degree in EE from the Helsinki University of Technology (TKK) (now Aalto University), Espoo, Finland, in 1981. In 1989, he was appointed Professor Chair of Radio Engineering with TKK, after holding the same position pro tem in 1985 and 19871989. He has been a Visiting Scientist and Professor with the Five College Radio Astronomy Observatory (FCRAO) and the University of Massachusetts at Amherst (1978-1979, 1980, 1981); the Chalmers University of Technology (1983); the University of California at Berkeley (1984-1985); the Jet Propulsion Laboratory (JPL) and California Institute of Technology (1992-1993); Observatoire de Paris and Universite de Paris 6 (2001-2002), and Universidad Carlos III de Madrid (2013-2014).

He supervises research in millimeter-wave components, antennas, receivers, microwave measurements, etc., at the Aalto University, Department of Radio Science and Engineering and Millimetre Wave Laboratory of Finland - ESA External Laboratory (MilliLab). He has authored or coauthored over 500 scientific or technical papers and seven books, e.g., Semiconductor Terahertz Technology: Devices and Systems at Room Temperature Operation (Wiley, 2015)

Dr. Räisänen is Fellow of the Antenna Measurement Techniques Association (AMTA) since 2008. He was the recipient of the AMTA Distinguished Achievement Award in 2009. The Chair of Excellence by the University Carlos III of Madrid was awarded to him in 2013. He has been Conference Chairman of several international microwave and millimeter-wave conferences including the 1992 EuMC, and is serving as General Chair of the 2016 Global Symposium on Millimeter Waves. He was an Associate Editor of the IEEE Transactions on Microwave Theory and Techniques (2002-2005). He was a member of the Board of Directors of the European Microwave Association (EuMA) (2006-2011). He is currently Head of the Department of Radio Science and Engineering and Chair of the Board of Directors, MilliLab. 